\title{
The Role of Adjuvant Chemotherapy for Esophageal Squamous Cell Carcinoma Patients with Pathological Positive Lymph Nodes After Neoadjuvant Chemotherapy Followed by Esophagectomy: a Single Institute Retrospective Analysis
}

\author{
Takeharu Imai $^{1}$ (D) $\cdot$ Yoshihiro Tanaka $^{1}$ (D) $\cdot$ Yuta Sato $^{1} \cdot$ Junichi Mase $^{1} \cdot$ Tomonari Suetsugu $^{1} \cdot$ Masahiro Fukada $^{1}$. \\ Itaru Yasufuku ${ }^{1}$ - Yoshinori Iwata ${ }^{1}$. Ryutaro Mori ${ }^{1}$. Hisashi Imai ${ }^{1} \cdot$ Takazumi Kato $^{1} \cdot$ Naoki Okumura ${ }^{1}$. \\ Nobuhisa Matsuhashi ${ }^{1} \cdot$ Takao Takahashi $^{1} \cdot$ Manabu Futamura ${ }^{1} \cdot$ Kazuhiro Yoshida $^{1}$
}

Received: 14 December 2020 / Accepted: 9 August 2021 / Published online: 28 August 2021

(C) Indian Association of Surgical Oncology 2021

\begin{abstract}
The need for adjuvant therapy after radical resection for patients with stage II-III thoracic esophageal squamous cell carcinoma (TESCC) who have undergone neoadjuvant chemotherapy (NAC) has not been determined. Since recurrence can occur after radical resection and since the prognosis is still poor, it is necessary to consider additional treatment strategies, including adjuvant chemotherapy. We retrospectively investigated the significance of adjuvant therapy after NAC followed by radical resection for TESCC. Between 2008 and 2018, 115 patients with clinical stage II-III underwent radical subtotal esophagectomy after neoadjuvant therapy. Among them, 62 were analyzed, excluding patients with T4 tumors and patients who had undergone R plus resection or who were receiving preoperative chemoradiotherapy. We compared patients who received adjuvant chemotherapy with those who only received observation; we examined overall survival (OS) and recurrence rates. Twenty-nine patients (46.7\%) had lymph node metastasis, 12 of whom received adjuvant chemotherapy $(41.3 \%)$. The recurrence rates for patients with and without lymph node metastasis were $55.1 \%$ and $15.1 \%$, respectively $(p=0.0022)$. Among patients with lymph node metastasis, there was no significant difference in the recurrence rate $(p=0.9270)$ or OS $(p=0.5416)$ based on the administration of adjuvant chemotherapy. However, in 15 patients with two or more positive lymph nodes, adjuvant chemotherapy increased OS $(p=0.0404)$. Adjuvant chemotherapy was associated with improved OS in clinical stage II-III TESCC patients with two or more pathological positive lymph nodes after NAC followed by radical surgery.
\end{abstract}

Keywords Advanced esophageal cancer $\cdot$ Adjuvant therapy $\cdot$ After surgery $\cdot$ After neoadjuvant chemotherapy

\section{Introduction}

There are many treatment strategies for stage II-III resectable advanced esophageal cancer. Surgery alone is thought to lead to recurrence; moreover, the prognosis is poor. Therefore, identifying whether further treatment, including chemotherapy and chemoradiotherapy (CRT), should be performed preoperatively or postoperatively, and what regimen should be administrated is vital to improving patient outcomes. Based on the Japan Clinical Oncology Group

Kazuhiro Yoshida

kyoshida@gifu-u.ac.jp

1 Department of Surgical Oncology, Graduate School of Medicine, Gifu University, 1-1 Yanagido, Gifu 501-1194, Japan
(JCOG) 9204 trial, it is common in Japan to administer postoperative chemotherapy to prevent recurrence after radical resection [1]. In addition, following the results of the JCOG9907 trial, preoperative chemotherapy has become standard in the last 10 years $[2,3]$. Although the Japanese esophageal cancer treatment guidelines recommend 5fluorouracil and cisplatin (FP) therapy as preoperative chemotherapy, various regimens are administered. In our department, we administer biweekly docetaxel, cisplatin, and 5-fluorouracil (Bi-DCF) and docetaxel, nedaplatin, and S1 (DGS) as NAC; high response and disease control rates and a low rate of adverse events have been observed $[4,5]$. However, some patients still experience recurrence after NAC and R0 surgery; thus, it is necessary to consider further strengthening NAC regimens as well as the need for postoperative adjuvant therapy. 
Several studies have reported that postoperative chemotherapy or CRT improves the survival of patients with advanced esophageal cancer who do not receive preoperative therapy [6-9]. However, the need for adjuvant therapy for patients with stage II-III esophageal cancer who receive neoadjuvant chemotherapy (NAC) has not been determined. Due to the lack of large prospective controlled trials, the Japanese treatment guidelines "weakly recommend not to use adjuvant chemotherapy" after NAC and R0 surgery [3]. Conversely, the current National Comprehensive Cancer Network guidelines recommend either observation or adjuvant chemotherapy for patients with esophageal adenocarcinoma who have undergone preoperative CRT and surgery [10]. Therefore, the role of adjuvant therapy (including chemotherapy and CRT) after NAC and R0 esophagectomy is unclear.

Until now, our department has considered patients with pathological lymph node metastasis who undergo NAC and R0 surgery as candidates for postoperative adjuvant therapy. The objective of the present study was to assess the significance of adjuvant therapy after NAC and R0 surgery for clinical stage II or III advanced thoracic esophageal squamous cell carcinoma (TESCC).

\section{Materials and Methods}

\section{Patients}

Between January 2008 and December 2018, 115 patients with clinical stage II or III TESCC underwent subtotal esophagectomy at the Gifu University Hospital. Of them, 53 were excluded due to the following reasons: clinical T4 tumor, $n=35$; underwent non-radical surgery, $n=7$; received preoperative CRT, $n=16$ (with overlaps). Consequently, 62 patients with TESCC (12 patients with stage II disease and 50 patients with stage III disease) who underwent radical subtotal esophagectomy were enrolled (Supplemental Figure 1). All patients were staged preoperatively after esophagogastroduodenoscopy via biopsies and computed tomography according to the 7th edition of the American Joint Committee on Cancer Tumor-NodeMetastasis Classification.

\section{Outcomes}

We retrospectively assessed the relationship between adjuvant therapy after NAC followed by radical surgery and prognosis. The primary endpoint was overall survival (OS). The secondary endpoints were the recurrence rate, recurrence-free survival (RFS), and adverse events of adjuvant chemotherapy. Adverse events were graded according to the Common Terminology Criteria for Adverse Events (version 4.0; Japanese edition, JCOG version) [11]. Clinical data were collected from a prospectively maintained database of patients at the Gifu University Hospital. Postoperative complications were classified according to the Clavien-Dindo classification [12].

\section{Neoadjuvant and Adjuvant Chemotherapy}

All patients were treated in accordance with the Japanese Esophageal Cancer Treatment Guidelines. Two courses of Bi-DCF, DCF, DGS, or FP were administered $[4,5]$. The Bi-DCF regimen consisted of docetaxel $\left(35 \mathrm{mg} / \mathrm{m}^{2}\right)$ with cisplatin $\left(40 \mathrm{mg} / \mathrm{m}^{2}\right)$ on days 1 and 15 and 5 -fluorouracil (750 $\mathrm{mg} / \mathrm{m}^{2}$ ) on days $1-5$ and $15-19$, repeated every 4 weeks. The DCF regimen consisted of docetaxel $\left(70 \mathrm{mg} / \mathrm{m}^{2}\right)$ with cisplatin $\left(70 \mathrm{mg} / \mathrm{m}^{2}\right)$ on days 1,22 , and 43 and 5-fluorouracil $(750$ $\mathrm{mg} / \mathrm{m}^{2}$ ) on days $1-5,22-26$, and $43-47$. The DGS regimen consisted of docetaxel $\left(35 \mathrm{mg} / \mathrm{m}^{2}\right)$ with nedaplatin $(40 \mathrm{mg} /$ $\left.\mathrm{m}^{2}\right)$ on day 8 and $\mathrm{S} 1\left(80 \mathrm{mg} / \mathrm{m}^{2}\right)$ on days $1-14$, repeated every 4 weeks. The FP regimen consisted of cisplatin $\left(80 \mathrm{mg} / \mathrm{m}^{2}\right)$ on day 1 and 5-fluorouracil $\left(800 \mathrm{mg} / \mathrm{m}^{2}\right)$ on days $1-5$, repeated every 4 weeks. After the second cycle of NAC, we assessed the tumor response according to the Response Evaluation Criteria in Solid Tumors [13]. Patients underwent radical esophageal resection and lymphadenectomy 3-4 weeks after the completion of chemotherapy.

Patients received biweekly DCF, DGS, or FP for adjuvant chemotherapy. The details of each regimen were the same as those for NAC. We recommend adjuvant chemotherapy for patients with pathological lymph node metastasis. However, because patients are maximizing their physical fitness with NAC and surgery, they decide whether to receive adjuvant chemotherapy after being adequately informed. Most patients are discharged within 1 month after surgery; we try to start adjuvant chemotherapy within 2 months after surgery.

\section{Surgery and Perioperative Management}

Nutrition management, speech exercise therapy, and oral care between NAC and surgery are vital [14]. Beginning 1 week before surgery, an immunoboost containing arginine was administered. Just before surgery, all patients received prophylactic antibiotics and methylprednisolone ( $250 \mathrm{mg} /$ person). Differential lung ventilation was used during thoracotomy or thoracoscopic surgery. After surgery, the patient entered the intensive care unit (ICU), and their breathing was managed with a ventilator. Extubation was routinely performed and ambulation began on postoperative day (POD) 1 . The patient left the ICU, and enteral feeding was started on POD 2. Swallowing fluoroscopy using gastrografin was performed on POD 7, and swallowing training with jelly meal was started. Patients were discharged if they were able to consume more than half of the postoperative meal. Before being discharged from the hospital, enteral nutrition via a jejunostomy tube was intermittently administered twice a 
day and was continued at home for 3 to 6 months after surgery.

\section{Surgical Procedure for Transthoracic Esophagectomy}

Subtotal esophagectomy using the thoracic approach is standard for stage II-III TESCC. Three-field dissection was performed when the tumor was in the upper or middle thoracic esophagus. We routinely performed dissection around the bilateral recurrent laryngeal nerves from the upper mediastinal lymph nodes to the cervical lymph nodes as caudally as possible. When the tumor was in the lower or abdominal esophagus, two-field dissection was performed. However, if the intraoperative rapid histopathological diagnosis of the lymph nodes around the recurrent laryngeal nerves indicated metastasis, three-field dissection was performed. We usually perform reconstruction using the subtotal stomach through the posterior mediastinal route [15]. However, the retrosternal route was selected when radiotherapy to the posterior mediastinum was considered because of locally advanced cancer. When the stomach could not be used for reconstruction after gastrectomy, reconstruction was performed using the ileum and right colon. We routinely perform jejunostomy to allow for postoperative enteral nutrition.

\section{Statistical Analysis}

Results are expressed as median (interquartile range) for quantitative variables and number (percentage) for qualitative variables. The $\chi 2$ test or Fisher's exact test was used for categorical variables, while the nonparametric Wilcoxon rank sum test was used for continuous variables. $p$ Values $<0.05$ were considered statistically significant. The Kaplan-Meier method was used to estimate survival, and the log-rank test was used to determine significant differences in survival. All statistical analyses were performed using JMP14 (SAS Institute Inc., Cary, NC, USA).

\section{Results}

\section{Patient Characteristics}

Supplemental Table 1 shows the clinical characteristics of the 62 enrolled patients. The median age was 67 years (range, 3685 years), and the majority of patients were men $(87 \%)$. Most tumors were located in the middle or lower thoracic esophagus (88.7\%), and 50 patients had clinical stage III disease (80.6\%). Bi-DCF $(58.0 \%)$ and DGS (29.0\%) were the most common $\mathrm{NAC}$ regimens. The preoperative response rate for NAC was $88.7 \%$, and the disease control rate was $98.3 \%$.

\section{Surgical Characteristics and Outcomes}

Supplemental Table 1 shows the surgical characteristics and postoperative complications of all 62 patients. Forty-seven patients $(75.8 \%)$ underwent thoracoscopic subtotal esophagectomy, and 28 patients $(45.1 \%)$ underwent three-field lymphadenectomy. Most patients underwent reconstruction using the subtotal stomach $(91.9 \%)$ thorough the posterior mediastinal route $(87.0 \%)$.

\section{Postoperative Complications}

Supplemental Table 1 shows the postoperative complications of 17 patients (27.4\%; grade II or higher). There were no cases of anastomotic leakage or pneumonia.

\section{Pathological Findings and Survival Rate}

The median follow-up time was 37.8 months. Twenty-nine patients had pathological positive lymph node metastasis (46.7\%); the recurrence rate for these patients was $55.1 \%$, whereas it was $15.1 \%$ for patients without lymph node metastasis $(p=0.0022)$. The prognosis of patients with pathological lymph node metastasis was significantly poorer than that of patients without lymph node metastasis (Supplemental Figure 2). The median RFS for patients with lymph node metastasis was 18.5 months, whereas it was not reached in patients without lymph node metastasis $(p<0.0001$; Supplemental Figure 2a). Similarly, the median OS for patients with lymph node metastasis was 47.7 months, whereas it was not reached for patients without lymph node metastasis $(p=0.0072$; Supplemental Figure 2b).

\section{Postoperative Adjuvant Chemotherapy and Survival Rate}

Among 29 patients with pathological lymph node metastasis, 12 received adjuvant chemotherapy $(41.3 \%)$. No patients without pathological lymph node metastasis received adjuvant chemotherapy (Supplemental Figure 1).

Table 1 shows the characteristics of the 29 node-positive patients. Patients with more positive nodes were more likely to receive adjuvant chemotherapy; however, this finding was not significant ( $p=0.0641)$. There were no significant differences between which group in the postoperative performance status or prealbumin levels.

Table 2 shows the adjuvant therapy regimen and adverse events for the 12 patients with node-positive esophageal cancer (grade $\geq 3$ ) who received adjuvant therapy. Five (42\%), five ( $42 \%)$, and two (16\%) patients received FP, DGS, and Bi$\mathrm{DCF}$, respectively. One patient in each treatment group developed grade $\geq 3$ leukopenia and hyponatremia, and one patient 
Table 1 Characteristics in 29 patients with pathological nodepositive esophageal cancer
Fig. 1 Survival rate based on the administration of adjuvant chemotherapy in TESCC patients with lymph node metastasis $(\mathbf{a}, \mathbf{b})$ and in patients with two or more pathological positive lymph nodes (c, d). a RFS: median survival time (MST) with adjuvant chemotherapy, 37.7 months vs without adjuvant chemotherapy, 11.6 months. b OS after surgery: MST with adjuvant chemotherapy, not reached vs without adjuvant chemotherapy, 47.7 months. c RFS: median survival time (MST) with adjuvant chemotherapy, 37.7 months vs without adjuvant chemotherapy, 5.1 months. d OS after surgery: MST with adjuvant chemotherapy, not reached vs without adjuvant chemotherapy, 20.1 months. TESCC, thoracic esophageal squamous cell carcinoma; RFS, recurrencefree survival; OS, overall survival

\begin{tabular}{|c|c|c|c|c|}
\hline & & \multicolumn{2}{|c|}{ Adjuvant chemotherapy } & \multirow[t]{2}{*}{$p$} \\
\hline & & (+) $n=12$ & (-) $n=17$ & \\
\hline \multirow[t]{2}{*}{ Age } & $<70$ years & 9 & 9 & 0.2731 \\
\hline & $\geq 70$ years & 3 & 8 & \\
\hline \multirow[t]{2}{*}{ Gender } & Male & 12 & 14 & 0.2463 \\
\hline & Female & 0 & 3 & \\
\hline \multirow[t]{3}{*}{ NAC regimen } & DGS & 4 & 4 & 0.0532 \\
\hline & DCF & 3 & 0 & \\
\hline & Bi-DCF & 5 & 13 & \\
\hline \multirow[t]{2}{*}{ Postoperative PS } & 1 & 12 & 15 & 0.4975 \\
\hline & 2 & 0 & 2 & \\
\hline \multirow[t]{2}{*}{ Prealbumin levels after surgery } & Median (mg/dL) & 20.5 & 22 & 0.5632 \\
\hline & [range] & [16-39] & [15-28] & \\
\hline \multirow[t]{2}{*}{ Tumor location } & $\mathrm{Ut} / \mathrm{Mt}$ & 8 & 13 & 0.8728 \\
\hline & $\mathrm{Lt}$ & 4 & 4 & \\
\hline \multirow[t]{2}{*}{$\mathrm{p}$ T classification } & $0 / 1$ & 3 & 5 & $>0.99$ \\
\hline & $2 / 3$ & 9 & 12 & \\
\hline \multirow[t]{2}{*}{$\mathrm{p} N$ classification } & 1 & 2 & 9 & 0.0641 \\
\hline & $2 / 3$ & 10 & 8 & \\
\hline \multirow[t]{2}{*}{ p stage } & II & 2 & 8 & 0.1260 \\
\hline & III & 10 & 9 & \\
\hline \multirow[t]{2}{*}{ CT grade } & $1 \mathrm{a} / 1 \mathrm{~b}$ & 10 & 13 & $>0.99$ \\
\hline & $2 / 3$ & 2 & 4 & \\
\hline
\end{tabular}

$N A C$ neoadjuvant chemotherapy; $U t$ upper thoracic esophagus; $M t$ middle thoracic esophagus; $L t$ lower thoracic esophagus; $B i-D C F$ biweekly docetaxel, cisplatin, and 5-fluorouracil; $D C F$ docetaxel, cisplatin, and 5fluorouracil; DGS docetaxel, nedaplatin, and S1; PS performance status; $p$ pathological; $C T$ grade pathological effect grade of NAC

a

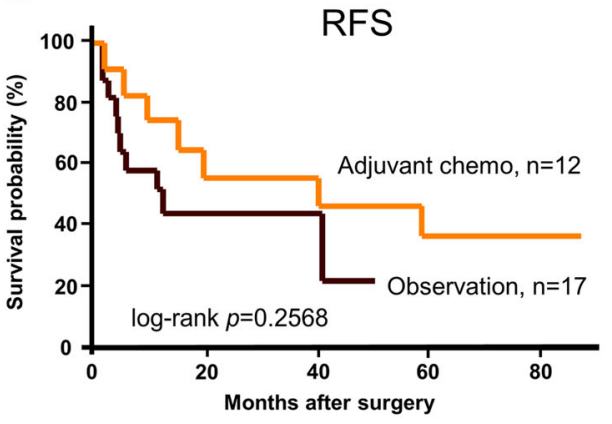

C

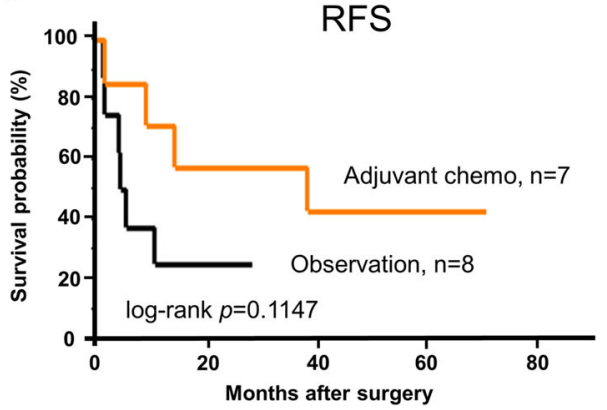

b

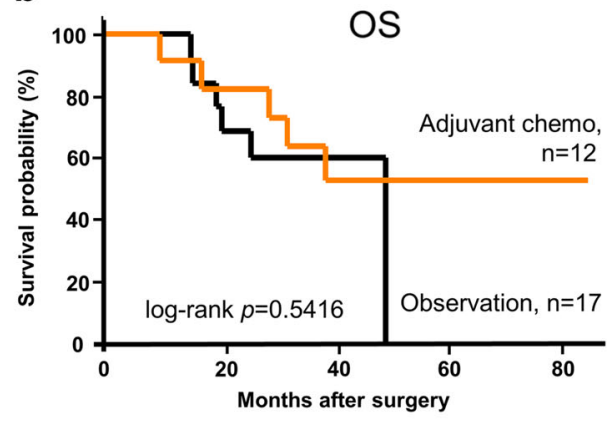

d

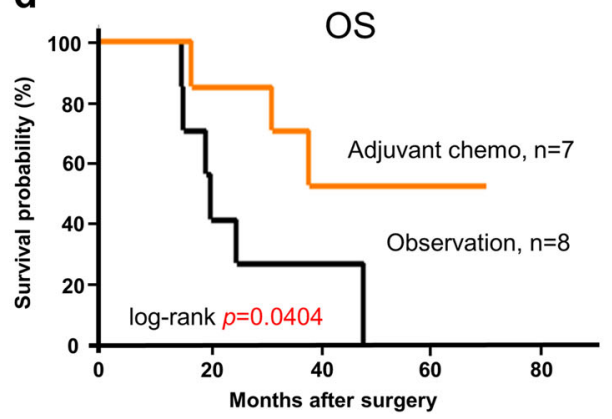


(8.3\%) developed grade $\geq 3$ anorexia. There were no chemotherapy-related deaths.

Supplemental Table 2 shows the recurrence rate and metastatic details of the 29 patients with pathological node-positive esophageal cancer. The recurrence rates for patients who did and did not receive adjuvant chemotherapy were $50.0 \%$ and $58.8 \%$, respectively $(p=0.9270)$. There was no statistically significant difference in the number of distant metastases between patients who received adjuvant chemotherapy and those who did not $(83.3 \%$ vs $40.0 \%, p=0.1451)$.

Table 3 shows the correlation between recurrence and clinicopathological factors in 29 patients with pathological nodepositive ESCC. Recurrence was significantly correlated with $\operatorname{sex}(p=0.0301)$ and venous invasion $(p=0.0260)$.

Figure 1 shows the postoperative survival rates in 29 patients with node-positive esophageal cancer. The median RFS in patients who received adjuvant chemotherapy was 37.7 months, whereas it was 11.6 months in patients who did not receive adjuvant chemotherapy (log-rank test, $p=0.2568$; Figure 1a). The median OS for patients who received adjuvant chemotherapy was reached, whereas it was 47.7 months for patients who did not receive adjuvant chemotherapy (log-rank test, $p=0.5416$; Figure $1 \mathrm{~b}$ ).

Figure 1 also shows the postoperative survival rates of 15 patients with two or more pathological positive lymph nodes. There was no significant difference in RFS based on the administration of adjuvant chemotherapy among these patients (no chemotherapy, 5.1 months; chemotherapy, 37.7 months;
Table 3 Correlation between recurrence and clinicopathological factors in 29 TESCC patients with lymph node metastasis

\begin{tabular}{|c|c|c|c|c|}
\hline & & \multicolumn{2}{|l|}{ Recurrence } & \multirow[t]{2}{*}{$p$} \\
\hline & & (+) $n=16$ & (-) $n=13$ & \\
\hline \multirow[t]{2}{*}{ Age } & $<70$ years & 9 & 9 & \multirow[t]{2}{*}{0.7401} \\
\hline & $\geq 70$ years & 7 & 4 & \\
\hline \multirow[t]{2}{*}{ Gender } & Male & 16 & 9 & \multirow[t]{2}{*}{0.0301} \\
\hline & Female & 0 & 4 & \\
\hline \multirow[t]{2}{*}{ Postoperative PS } & 1 & 16 & 11 & \multirow[t]{2}{*}{0.1921} \\
\hline & 2 & 0 & 2 & \\
\hline \multirow[t]{2}{*}{ Tumor location } & $\mathrm{Ut} / \mathrm{Mt}$ & 13 & 8 & \multirow[t]{2}{*}{0.4057} \\
\hline & $\mathrm{Lt}$ & 3 & 5 & \\
\hline \multirow[t]{2}{*}{ pT classification } & $0 / 1$ & 6 & 2 & \multirow[t]{2}{*}{0.2378} \\
\hline & $2 / 3$ & 10 & 11 & \\
\hline \multirow[t]{2}{*}{$\mathrm{pN}$ classification } & 1 & 6 & 5 & \multirow[t]{2}{*}{0.7401} \\
\hline & $2 / 3$ & 10 & 8 & \\
\hline \multirow[t]{2}{*}{ p stage } & II & 7 & 3 & \multirow[t]{2}{*}{0.4334} \\
\hline & III & 9 & 10 & \\
\hline \multirow[t]{2}{*}{ Differentiation } & Well & 6 & 6 & \multirow[t]{2}{*}{0.9270} \\
\hline & Moderate/poor & 10 & 7 & \\
\hline \multirow[t]{2}{*}{ Lymphatic invasion } & - & 0 & 2 & \multirow[t]{2}{*}{0.1921} \\
\hline & + & 16 & 11 & \\
\hline \multirow[t]{2}{*}{ Venous invasion } & - & 1 & 6 & \multirow[t]{2}{*}{0.0260} \\
\hline & + & 15 & 7 & \\
\hline \multirow[t]{2}{*}{ CT grade } & $1 \mathrm{a} / 1 \mathrm{~b}$ & 15 & 8 & \multirow[t]{2}{*}{0.0638} \\
\hline & $2 / 3$ & 1 & 5 & \\
\hline
\end{tabular}

TESCC thoracic esophageal squamous cell carcinoma, $P S$ performance status, $U t$ upper thoracic esophagus, $M t$ middle thoracic esophagus, $L t$ lower thoracic esophagus, $p$ pathological, $C T$ grade pathological effect grade of neoadjuvant chemotherapy
Table 2 Adjuvant therapy for 12 patients with node-positive esophageal cancer and grade $\geq 3$ adverse events

\begin{tabular}{ll}
\hline & $n(\%)$ \\
\hline Adjuvant chemotherapy, regimen, and dose & $5(42)$ \\
FP & 5 \\
5-FU: $800 \mathrm{mg} / \mathrm{m}^{2}$, CDDP: $80 \mathrm{mg} / \mathrm{m}^{2}(100 \%$ dose $), 2$ courses & $5(42)$ \\
DGS & 1 \\
S-1: $80 \mathrm{mg} /$ body, CDGP: $40 \mathrm{mg} / \mathrm{m}^{2}$, TXT: $35 \mathrm{mg} / \mathrm{m}^{2}(100 \%$ dose $), 2$ courses & 1 \\
S-1: $80 \mathrm{mg} /$ body, CDGP: $36 \mathrm{mg} / \mathrm{m}^{2}$, TXT: $31.5 \mathrm{mg} / \mathrm{m}^{2}(90 \%$ dose $), 2$ courses & 3 \\
S-1: $80 \mathrm{mg} /$ body, CDGP: $32 \mathrm{mg} / \mathrm{m}^{2}$, TXT: $28 \mathrm{mg} / \mathrm{m}^{2}(80 \%$ dose $), 2$ courses & $1(8)$ \\
Bi-DCF & 1 \\
TXT: $31.5 \mathrm{mg} / \mathrm{m}$, CDDP: $36 \mathrm{mg} / \mathrm{m}^{2}, 5$-FU: $360 \mathrm{mg} / \mathrm{m}^{2}(90 \%$ dose $), 1$ course & $1(8)$ \\
wPTX & 1 \\
PTX: $80 \mathrm{mg} /$ body, 3 courses & 55 [45-93] \\
Time to start adjuvant chemotherapy after surgery & \\
Median [range], days & $1(8.3)$ \\
Grade $\geq 3$ adverse events & $1(8.3)$ \\
Hematologic toxicity & $1(8.3)$ \\
Leukopenia & \\
Hyponatremia &
\end{tabular}

5-FU 5-Fluorouracil, $C D D P$ cisplatin, $C D G P$ nedaplatin, $T X T$ docetaxel, $w P T X$ weekly pacritaxel 
log-rank test, $p=0.1147$; Figure 1c). However, there was a significant difference in OS (no chemotherapy, 20.1 months; chemotherapy, not reached; log-rank test, $p=0.0404$; Figure 1d). There were no significant differences in RFS or OS in 14 patients with only one pathological positive lymph node based on the administration of adjuvant chemotherapy (data not shown).

\section{Discussion}

Preoperative CRT followed by surgery is the most common treatment for patients with resectable advanced esophageal cancer, particularly in Western countries [16, 17]. However, in Japan where adenocarcinomas of the lower esophagus and esophagogastric junction are more prevalent than in Western countries, squamous cell carcinoma of the thoracic esophagus is the most common type of esophageal cancer. Traditionally, subtotal esophagectomy and three-field lymphadenectomy, including cervical lymphadenectomy, have been standard in Japan [18]. Additionally, to prevent recurrence after surgery, NAC rather than postoperative chemotherapy is administered in Japan, based on clinical trial results. The JCOG9204 trial showed that the 5-year OS rate was $52 \%$ with surgery alone and $61 \%$ with surgery plus chemotherapy including two courses of FP [1]. Because of these results, postoperative chemotherapy with FP was previously the standard treatment for patients with stage II-III squamous cell carcinoma in Japan. More recently, the JCOG9907 trial showed a 5-year OS rate of $43 \%$ in the postoperative chemotherapy group and $55 \%$ in the preoperative chemotherapy group [2]. Therefore, preoperative adjuvant chemotherapy ("NAC") has become the standard treatment for patients with stage II-III resectable esophageal squamous cell carcinoma. According to the subgroup analysis based on the depth of invasion, progression or recurrence after surgery was more common in cT3 cases (35\%) than in cT1-2 cases $(29 \%)$ among patients who received NAC. Therefore, more intense NAC is needed to control recurrence or distant metastasis. A new clinical trial (JCOG1109) is underway to examine the optimal NAC regimen to further improve prognosis [19]. Specifically, the aim of this trial is to confirm the superiority of DCF over FP and the superiority of FP with RT over FP alone as preoperative therapy for ESCC.

The V325 study group results suggest that a triplecombination regimen such as DCF is most effective for advanced esophageal cancer [20]. Therefore, a triplecombination regimen is used for patients with more advanced esophageal cancer. However, the DCF regimen also causes adverse events. In a phase I/II study of Bi-DCF for advanced ESCC $[4,5]$, although the prognosis was improved by NAC, the recurrence rate of FP therapy was high. Unsurprisingly, recurrence was also found to occur after Bi-DCF therapy. The development of perioperative chemotherapy with immune checkpoint inhibitors is also expected to progress. However, we believe it is necessary to administer intensive postoperative adjuvant therapy for patients with lymph node metastasis to the extent that it is tolerated. Therefore, in this study, we examined the utility of providing adjuvant chemotherapy after NAC plus R0 surgery to address the limitations of current treatments.

Previous studies have shown that postoperative therapies (including chemotherapy or CRT) after radical esophagectomy for esophageal cancer can improve survival, even without the administration of neoadjuvant chemotherapy [6-9, 21-23]. Postoperative radiotherapy was found to significantly increase disease-free survival and decreased local regional recurrence in patients with pathological T2-3N0M0 thoracic esophageal squamous cell carcinoma with acceptable toxicities [6]. In addition, postoperative adjuvant chemotherapy improves prognosis in patients with clinical stage II-III esophageal cancer [7] and is strongly associated with improved OS and disease-free survival in patients with pT3N0M0 TESCC [8]. Postoperative chemoradiotherapy mainly benefits patients with stage III disease, those with $<15$ resected lymph nodes, younger patients ( $<60$ years), and smokers with stage II-III TESCC [21]. Rucker et al. determined that the risks and benefits of adjuvant therapy should be weighed before offering it to patients with completely resected pT2-4aN0M0 esophageal adenocarcinoma [22]. Moreover, Li et al. reported that patients with fewer total resected lymph nodes and a higher lymph nodes ratio who underwent adjuvant therapy had significantly better survival than those who did not receive adjuvant therapy [9]. Therefore, stage II-III TESCC patients could benefit from postoperative CRT with manageable toxicities [23].

To date, a few studies have examined postoperative adjuvant therapy after NAC plus R0 surgery, which was the purpose of our study (Table 4). Drake et al. reported that adjuvant chemotherapy was associated with significantly improved survival in patients with node-positive esophageal adenocarcinoma after neoadjuvant therapy and complete resection [24]. Moreover, Semenkovich et al. reported that adjuvant treatment was independently associated with improved survival, with a $24 \%$ reduction in mortality in esophageal adenocarcinoma and squamous cell carcinoma patients with persistent node-positive disease after induction therapy and esophagectomy [25]. In contrast, Yan et al. reported that adjuvant chemotherapy did not influence disease-free survival or OS in patients with locally advanced esophageal squamous cell carcinoma after neoadjuvant platinum-based chemotherapy and surgery [26]. Our study focused on patients with thoracic esophageal squamous cell carcinoma who underwent esophagectomy after NAC, excluding patients with cervical esophageal cancer and esophagogastric junctional cancer. We found that (1) TESCC patients with pathological positive lymph nodes had higher recurrence rate after surgery and (2) adjuvant 
Table 4 Summary of outcomes for postoperative therapy in patients with esophageal cancer after neoadjuvant therapy and surgery

\begin{tabular}{|c|c|c|c|c|c|c|}
\hline Reference (first author) & Year & Histology & Neoadjuvant treatment & Adjuvant treatment & OS & $p$ \\
\hline Drake et al. [24] & 2019 & $\mathrm{AC}$ & $\mathrm{CT}$ & CT/observation & $\begin{array}{l}\text { Median } \\
31.2 \mathrm{M} / 24.0 \mathrm{M}\end{array}$ & 0.031 \\
\hline Semenkovich et al. [25] & 2019 & $\mathrm{AC} / \mathrm{SCC}$ & RT & $\mathrm{CT}$ or CRT/observation & $\begin{array}{l}\text { Median } \\
2.6 \mathrm{Y} / 2.3 \mathrm{Y}\end{array}$ & 0.02 \\
\hline Yan et al. [26] & 2019 & SCC & $\mathrm{CT}$ & CT/observation & $\begin{array}{l}5-Y \text { OS } \\
62.4 \% / 68.6 \%\end{array}$ & 0.359 \\
\hline Our study $^{\mathrm{a}}$ & 2020 & SCC & $\mathrm{CT}$ & CT/observation & $\begin{array}{l}\text { Median } \\
\text { Not reached/20.1 M }\end{array}$ & 0.040 \\
\hline
\end{tabular}

$A C$ adenocarcinoma, $S C C$ squamous cell carcinoma, $C T$ chemotherapy, $R T$ radiotherapy, $C R T$ chemoradiotherapy, $M$ month, $Y$ year

${ }^{a}$ Thoracic esophageal cancer patients with two or more positive lymph nodes

chemotherapy was effective for patients with two or more positive lymph nodes. The tolerability of adjuvant therapy is a known problem; however, in all these reports, adjuvant therapy was safely administered. DGS was chosen in this study because it can be administered as outpatient chemotherapy, but it often required dose reduction. However, FP therapy could be administered without dose reduction. There were few adverse events (grade $\geq 3$ ) following adjuvant chemotherapy. Since we routinely perform enteral nutrition using a jejunostomy tube for 6 months after surgery [15], we believe that even patients who have completed NAC and surgery could safely receive postoperative adjuvant chemotherapy.

Finally, a recently published global, randomized phase III trial (CheckMate 577) demonstrated that nivolumab, a checkpoint inhibitor (ICI), has beneficial effects as an adjuvant therapy in patients with stage II or III esophageal or gastroesophageal junction cancer who have undergone R0 resection after receiving neoadjuvant chemoradiotherapy [27]. In addition, the grade 3 or 4 adverse events were acceptable. As in our study, some populations may require adjuvant therapy to prevent recurrence, even if they receive preoperative treatment. In the future, the development of adjuvant therapy using ICI is expected.

The limitations of our study are its retrospective design, the small, single institute cohort, and limited follow-up. Despite the lack of difference in performance status and prealbumin levels before adjuvant chemotherapy, this was not a prospective, randomized trial, and the two groups of patients were not the same. Furthermore, a median follow-up of $<37.9$ months is insufficient to make conclusions regarding long-term oncologic outcomes for TESCC patients. Therefore, randomized controlled studies are necessary to further evaluate the role of adjuvant chemotherapy after NAC and R0 esophagectomy for clinical stage II-III TESCC patients.

\section{Conclusion}

Postoperative adjuvant chemotherapy was associated with improved OS in clinical stage II-III TESCC patients with two or more pathological positive lymph nodes after NAC and radical esophagectomy.

Abbreviations TESCC, Thoracic esophageal squamous cell carcinoma; NAC, Neoadjuvant chemotherapy; OS, Overall survival; CRT, Chemoradiotherapy; JCOG, Japan Clinical Oncology Group; FP, 5Fluorouracil and cisplatin; Bi-DCF, Biweekly docetaxel, cisplatin, and 5-fluorouracil; DGS, Docetaxel, nedaplatin, and S1; POD, Postoperative day; ICU, Intensive care unit; RFS, Recurrence-free survival; LNs, Lymph nodes

Supplementary Information The online version contains supplementary material available at https://doi.org/10.1007/s13193-021-01419-0.

Acknowledgement We would like to thank Editage (www.editage.com) for English language editing.

Author Contribution TI and YT conceived the study and planned the study design as the principal investigators. TI interpreted the results and wrote the manuscript. YT and KY revised the manuscript draft by adding intellectual insights and provided critical advice. All other authors obtained the data and provided critical comments to improve the manuscript. All authors approved the final version for submission.

Data Availability The datasets used and analyzed in this article are available from the corresponding author on reasonable request.

\section{Declarations}

Ethics Approval This study was approved by the review board of the Gifu University Hospital (approval no. 2019-203).

Consent for participate Informed consent was obtained in the form of opt-out on the website for the publication of this article.

Conflict of Interest Dr. Yoshida reports receipt of grants, personal fees, and non-financial support from EA Pharma Co., Ltd., Sanofi, Yakult Honsha Co., Ltd., Chugai Pharmaceutical Co., Ltd., Taiho Pharmaceutical Co., Ltd., Takeda Pharmaceutical Co., Ltd., Eli Lilly Japan K.K., Daiichi Sankyo Co., Ltd., Ono Pharmaceutical Co., Ltd., Merck Serono Co., Ltd., and Novartis Pharma K.K. and grants from Kyowa Hakko Kirin Co., Ltd. outside of the submitted work. Dr. Tanaka reports receipt of grants from Daiichi Sankyo Co., Ltd. outside of the submitted work. Other authors have no conflict of interest to disclose. 


\section{References}

1. Ando N, Iizuka T, Ide H, Ishida K, Shinoda M, Nishimaki T, Takiyama W, Watanabe H, Isono K, Aoyama N, Makuuchi H, Tanaka O, Yamana H, Ikeuchi S, Kabuto T, Nagai K, Shimada Y, Kinjo Y, Fukuda H, Japan Clinical Oncology Group (2003) Surgery plus chemotherapy compared with surgery alone for localized squamous cell carcinoma of the thoracic esophagus: a Japan Clinical Oncology Group Study-JCOG9204. J Clin Oncol 21: 4592-4596. https://doi.org/10.1200/JCO.2003.12.095

2. Ando N, Hoichi K, Igaki H, Shinoda M, Ozawa S, Shimizu H, Nakamura T, Yabusaki H, Aoyama N, Kurita A, Ikeda K, Kanda T, Tsujinaka T, Nakamura K, Fukuda H (2012) A randomized trial comparing postoperative adjuvant chemotherapy with cisplatin and 5 -fluorouracil versus preoperative chemotherapy for localized advanced squamous cell carcinoma of the thoracic esophagus (JCOG9907). Ann Surg Oncol 19:68-74. https://doi.org/10.1245/ s10434-011-2049-9

3. Kitagawa Y, Uno T, Oyama T, Kato K, Kato H, Kawakubo H, Kawamura O, Kusano M, Kuwano H, Takeuchi H, Toh Y, Doki Y, Naomoto Y, Nemoto K, Booka E, Matsubara H, Miyazaki T, Muto M, Yanagisawa A, Yoshida M (2019) Esophageal cancer practice guidelines 2017 edited by the Japan esophageal society: Part 2. Esophagus 16:25-43. https://doi.org/10.1007/s10388-0180642-8

4. Tanaka Y, Yoshida K, Yamada A, Tanahashi T, Okumura N, Matsuhashi N, Yamaguchi K, Miyazaki T (2016) Phase II trial of biweekly docetaxel, cisplatin, and 5-fluorouracil chemotherapy for advanced esophageal squamous cell carcinoma. Cancer Chemother Pharmacol 77:1143-1152. https://doi.org/10.1007/s00280-0162985-y

5. Tanaka Y, Yoshida K, Tanahashi T, Okumura N, Matsuhashi N, Yamaguchi K (2016) Phase II trial of neoadjuvant chemotherapy with docetaxel, nedaplatin, and S1 for advanced esophageal squamous cell carcinoma. Cancer Sci 107:764-772. https://doi.org/10. 1111/cas.12943

6. Deng W, Yang J, Ni W, Li C, Chang X, Han W, Zhou Z, Chen D, Feng Q, Liang J, Lv J, Wang X, Wang X, Deng L, Wang W, Bi N, Zhang T, Li Y, Gao S et al (2020) Postoperative radiotherapy in pathological T2-3N0M0 thoracic esophageal squamous cell carcinoma: interim report of a prospective, phase III, randomized controlled study. Oncologist 25:e701-e708. https://doi.org/10.1634/ theoncologist.2019-0276

7. Sohda M, Saito H, Kuriyama K, Yoshida T, Kumakura Y, Honjyo H, Hara K, Ozawa D, Suzuki S, Tanaka N, Sakai M, Miyazaki T, Fukuchi M, Kuwano H (2019) Post-esophagectomy adjuvant chemotherapy benefits esophageal cancer patients. In Vivo 33:501506. https://doi.org/10.21873/invivo.11502

8. Wang Q, Peng L, Li T, Dai W, Jiang Y, Xie T, Fang Q, Wang Y, Wu L, Cao B, Han Y, Lang J (2020) Postoperative chemotherapy for thoracic pathological T3N0M0 esophageal squamous cell carcinoma. Ann Surg Oncol 27:1488-1495. https://doi.org/10.1245/ s10434-019-08112-1

9. Li Y, Zhao W, Ni J, Zou L, Yang X, Yu W, Fu X, Zhao K, Zhang Y, Chen H, Xiang J, Xie C, Zhu Z (2019) Predicting the value of adjuvant therapy in esophageal squamous cell carcinoma by combining the total number of examined lymph nodes with the positive lymph node ratio. Ann Surg Oncol 26:2367-2374. https://doi.org/ 10.1245/s10434-019-07489-3

10. NCCN guidelines version 1.2018 Esophageal and Esophagogastric Junction Cancers. (2018) https://www.nccn.org/professionals/ physician_gls/pdf/esophageal.pdf

11. Common terminology criteria for adverse events (CTCAE) version 4.03: published Jun.14, 2010
12. Dindo D, Demartines N, Clavien PA (2004) Classification of surgical complications: a new proposal with evaluation in a cohort of 6336 patients and results of a survey. Ann Surg 240:205-213. https://doi.org/10.1097/01.sla.0000133083.54934.ae

13. Eisenhauer EA, Therasse P, Bogaerts J, Schwartz LH, Sargent D, Ford R, Dancey J, Arbuck S, Gwyther S, Mooney M, Rubinstein L, Shankar L, Dodd L, Kaplan R, Lacombe D, Verweij J (2009) New response evaluation criteria in solid tumours: revised RECIST guideline (version 1.1). Eur J Cancer 45:228-247. https://doi.org/ 10.1016/j.ejca.2008.10.026

14. Tanaka Y, Takahashi T, Yamaguchi K, Osada S, Shimokawa T, Yoshida K (2016) Elemental diet plus glutamine for the prevention of mucositis in esophageal cancer patients receiving chemotherapy: a feasibility study. Support Care Cancer 24:933-941. https://doi. org/10.1007/s00520-015-2864-9

15. Yoshida K, Tanaka Y, Imai T, Sato Y, Hatanaka Y, Suetsugu T, Okumura N, Matsuhashi N, Takahashi T, Yamaguchi K (2020) Subtotal stomach in esophageal reconstruction surgery achieves an anastomotic leakage rate of less than $1 \%$. Ann Gastroenterol Surg 4:422-432. https://doi.org/10.1002/ags3.12336

16. Herskovic A, Martz K, al-Sarraf M, Leichman L, Brindle J, Vaitkevicius V, Cooper J, Byhardt R, Davis L, Emami B (1992) Combined chemotherapy and radiotherapy compared with radiotherapy alone in patients with cancer of the esophagus. N Engl $\mathrm{J}$ Med 326:1593-1598. https://doi.org/10.1056/ NEJM199206113262403

17. Shapiro J, van Lanschot JJB, Hulshof MCCM, van Hagen P, van Berge Henegouwen MI, Wijnhoven BPL, van Laarhoven HWM, Nieuwenhuijzen GAP, Hospers GAP, Bonenkamp JJ, Cuesta MA, Blaisse RJB, Busch ORC, Ten Kate FJW, Creemers GM, Punt CJA, Plukker JTM, Verheul HMW, Bilgen EJS et al (2015) Neoadjuvant chemoradiotherapy plus surgery versus surgery alone for oesophageal or junctional cancer (CROSS): long-term results of a randomised controlled trial. Lancet Oncol 16:1090-1098. https:// doi.org/10.1016/S1470-2045(15)00040-6

18. Udagawa $\mathrm{H}$ (2020) Past, present, and future of three-field lymphadenectomy for thoracic esophageal cancer. Ann Gastroenterol Surg 4:324-330. https://doi.org/10.1002/ags3.12338

19. Nakamura K, Kato K, Igaki H, Ito Y, Mizusawa J, Ando N, Udagawa H, Tsubosa Y, Daiko H, Hironaka S, Fukuda H, Kitagawa Y, Japan Esophageal Oncology Group/Japan Clinical Oncology Group (2013) Three-arm phase III trial comparing cisplatin plus 5-FU (CF) versus docetaxel, cisplatin plus 5-FU (DCF) versus radiotherapy with $\mathrm{CF}$ (CF-RT) as preoperative therapy for locally advanced esophageal cancer (JCOG1 109, NExT study). Jpn J Clin Oncol 43:752-755. https://doi.org/10.1093/jjco/hyt061

20. Ajani JA, Moiseyenko VM, Tjulandin S, Majlis A, Constenla M, Boni C, Rodrigues A, Fodor M, Chao Y, Voznyi E, Awad L, Van Cutsem E, V-325 Study Group (2007) Quality of life with docetaxel plus cisplatin and fluorouracil compared with cisplatin and fluorouracil from a phase III trial for advanced gastric or gastroesophageal adenocarcinoma: the V-325 Study Group. J Clin Oncol 25:3210 3216. https://doi.org/10.1200/JCO.2006.08.3956

21. Zou B, Tu Y, Liao D, Xu Y, Wang J, Huang M, Ren L, Zhu J, Gong Y, Liu Y, Zhou L, Zhou X, Peng F, Lu Y (2020) Radical esophagectomy for stage II and III thoracic esophageal squamous cell carcinoma followed by adjuvant radiotherapy with or without chemotherapy: which is more beneficial? Thorac Cancer 11:631-639. https://doi.org/10.1111/1759-7714.13307

22. Rucker AJ, Raman V, Jawitz OK, Voigt SL, Harpole DH, D'Amico TA, Tong BC (2020) The impact of adjuvant therapy on survival after esophagectomy for node-negative esophageal adenocarcinoma. Ann Surg. Publish Ahead of Print. https://doi.org/10.1097/ SLA.0000000000003886

23. Song T, Chen P, Fang M, Zhang X, Du D, Wu S (2020) The role of adjuvant chemoradiotherapy over radiotherapy after $\mathrm{R} 0$ resection 
for Stage II-III esophageal squamous cell carcinoma. Cancer Manag Res 12:1631-1639. https://doi.org/10.2147/CMAR. S232930

24. Drake J, Tauer K, Portnoy D, Weksler B (2019) Adjuvant chemotherapy is associated with improved survival in patients with nodal metastases after neoadjuvant therapy and esophagectomy. $\mathrm{J}$ Thorac Dis 11:2546-2554. https://doi.org/10.21037/jtd.2019.05.66

25. Semenkovich TR, Subramanian M, Yan Y, Hofstetter WL, Correa AM, Cassivi SD, Inra ML, Stiles BM, Altorki NK, Chang AC, Brescia AA, Darling GE, Allison F, Broderick SR, Etchill EW, Fernandez FG, Chihara RK, Litle VR, Muñoz-Largacha JA et al (2019) Adjuvant therapy for node-positive esophageal cancer after induction and surgery: a multisite study. Ann Thorac Surg 108: 828-836. https://doi.org/10.1016/j.athoracsur.2019.04.099
26. Wanpu Y, Zhao Peiliang FH, Lin Y, Li Z, Dai L, Yang Y, Kang X, Chen KN (2019) Survival after induction chemotherapy and esophagectomy is not improved by adjuvant chemotherapy. Ann Thorac Surg 108:1505-1513. https://doi.org/10.1016/j.athoracsur.2019.04. 106

27. Ronan JK, Jaffer AA, Jaroslaw K, Thomas Z, Eric VC, Guillaume P, Guillermo M, Josephine F, Satoru M, Astrid L, Hope U, Elena E, Cecile G, Karen G, Syed Z, Stephanie S, Andrew H, Kynan F, Michael S et al (2021) Adjuvant nivolumab in resected esophageal or gastroesophageal junction cancer. N Engl J Med 384:11911203. https://doi.org/10.1056/NEJMoa2032125

Publisher's Note Springer Nature remains neutral with regard to jurisdictional claims in published maps and institutional affiliations. 